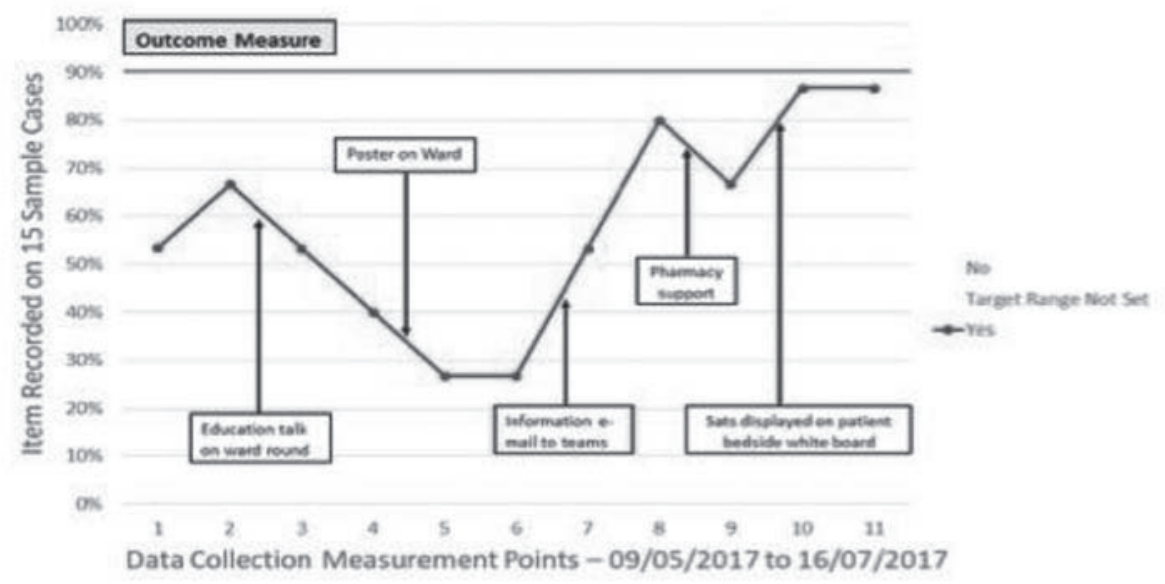

Abstract M9 Figure 1 Do oxygen saturations on observations chart match target saturations on drug chart?

\section{M10 DEMONSTRATING THE POTENTIAL ROLE OF COMMUNITY PHARMACISTS IN IMPROVING CARE OF COPD PATIENTS}

D Attar-Zadeh, A Guirguis, CE Heading, S Shah, U Shah, S Bancroft. London North West Local Practice Forum of Royal Pharmaceutical Society, London, UK

\subsection{6/thoraxjnl-2017-210983.432}

Introduction and Objectives Although there is some realisation of the potential for community pharmacists to help patients manage their conditions, finding ways to demonstrate this potential to health professionals in different roles and sectors is not easy. We conducted a semi-quantitative analysis of support offered to COPD patients within normal limits of practice in community pharmacies, with the intention of sharing our findings as widely as possible. These findings subsequently formed the basis of an infographic that can be distributed in a variety of scenarios.

Methods The study, in NW London, involved 18 pharmacies. Over a 4 week period in February-March 2015, pharmacists undertook consultations in the pharmacy with consenting patients who were receiving medicines prescribed for COPD. Patients were asked questions from a semi-structured questionnaire. Information was collected and action taken to provide high value interventions and referral, where appropriate. The collected data were analysed and key findings identified for sharing in an infographic.

Results At the consultation, of 135 patients, 56\% were provided with inhaler training, 65\% were offered Medicines Use Reviews, $17 \%$ received guidance regarding rescue packs, 28\% were referred to GPs and $82 \%$ of smokers $(n=39)$ were referred to stop smoking services. $84 \%$ of patients had received prior flu vaccination. Areas of clinical concern identified included poor inhaler technique, poor familiarity with pulmonary rehabilitation services, higher than expected ICS use and medication or other issues requiring referral to GPs (28\%). The ratio of men to women (1:0.7) was consistent with published data, but the ethnicity of patients did not match the pattern expected in the locality on the basis of Public Health and census information.
Conclusions The analysis yielded evidence of how community pharmacists can both assist in the management of individual patients with COPD, and provide a snapshot of support in a locality. Summarising this evidence as an infographic that can be distributed digitally, and at professional and educational meetings, may hasten recognition of the potential usefulness of this type of support and the value of community pharmacies as a resource. The approach will be applied to other conditions, subject to evaluation of effectiveness.

\section{M11 THE USE OF ASTHMA CARE BUNDLE PROFORMAS CAN IMPROVE QUALITY OF CARE IN ACUTE ASTHMA ADMISSIONS}

M Pace-Bardon, D Bilocca, K Jackson, P Bradding, RH Green. Glenfield Hospital, Leicester, UK

\subsection{6/thoraxjnl-2017-210983.433}

Introduction Despite the introduction of dedicated asthma services and targeted therapies, asthma exacerbations remain a common cause of hospital admission with significant utilisation of health care resources.

Aim To determine whether asthma care bundle proformas contribute to improved quality of care in adult patients admitted to Glenfield Hospital with an asthma exacerbation

Method Data collected at Glenfield hospital as part of the national BTS Adult Asthma audit in 2011 and 2012, prior to the introduction of asthma care bundles, was compared to data collected using a similar methodology in 2016, when both admission and discharge bundles had been introduced. The Results were analysed using Chi-squared Testing.

Results Asthma Care bundles were used in $64.4 \%$ of asthma admissions audited in 2016. When compared to 2011 and 2012, prior to the introduction of care bundles, there was a statistically significant increase in the proportion of patients having a documented peak flow on admission from $73.1 \%$ in $2011 / 2 \%$ to $94.4 \%$ in $2016(p=0.001)$. There was also an improvement in the frequency of inhaler technique assessment on discharge with an increase from $52.5 \%$ in $2011 / 2 \%$ to 